\title{
Evaluation of gantry speed on image quality and imaging dose for 4D cone- beam CT acquisition
}

Andrew P. Santoso ${ }^{1}$, Kwang H. Song ${ }^{2,3}$, Yujiao Qin ${ }^{3}$, Stephen J. Gardner ${ }^{3}$, Chang Liư ${ }^{3}$, Indrin J. Chetty ${ }^{3}$, Benjamin Movsas ${ }^{3}$, Munther Ajlouni ${ }^{3}$ and Ning Wen ${ }^{3 *}$

\begin{abstract}
Background: This study investigates the effect of gantry speed on $4 D C B C T$ image quality and dose for the Varian On-Board Imager ${ }^{\oplus}$.

Methods: A thoracic 4DCBCT protocol was designed using a $125 \mathrm{kVp}$ spectrum. Image quality parameters were evaluated for 4DCBCT acquisition using Catphan ${ }^{\circledR}$ phantom with real-time position management ${ }^{\mathrm{TM}}$ system for gantry speeds varying between 1.0 to $6.0 \%$ s. Superior-inferior motion of the phantom was executed using a sinusoidal waveform with five second period. Scans were retrospectively sorted into 4 phases (CBCT-4 ph) and 10 phases (CBCT-10 ph); average 4DCBCT (CBCT-ave), using all image data from the 4DCBCT acquisitions was also evaluated. The $4 \mathrm{DCBCT}$ images were evaluated using the following image quality metrics: spatial resolution, contrast-to-noise ratio (CNR), and uniformity index (UI). Additionally, Hounsfield unit (HU) sensitivity compared to a baseline CBCT and percent differences and RMS errors (RMSE) of excursion were also determined. Imaging dose was evaluated using an IBA CC13 ion chamber placed within CIRS Thorax phantom using the same sinusoidal motion and image acquisition settings as mentioned above.

Results: Spatial resolution decreased linearly from 5.93 to $3.82 \mathrm{lp} / \mathrm{cm}$ as gantry speed increased from 1.0 to $6.0^{\circ} / \mathrm{s}$. CNR decreased linearly from 4.80 to 1.82 with gantry speed increasing from 1.0 to $6.0 \%$, respectively. No noteworthy variations in $\mathrm{UI}, \mathrm{HU}$ sensitivity, or excursion metrics were observed with changes in gantry speed. Ion chamber dose rates measured ranged from 2.30 (lung) to 5.18 (bone) E-3 cGy/mAs.

Conclusions: A quantitative analysis of the Varian OBI's 4DCBCT capabilities was explored. Changing gantry speed changes the number of projections used for reconstruction, affecting both image quality and imaging dose if $x$-ray tube current is held constant. From the results of this study, a gantry speed between 2 and $3 \% \mathrm{~s}$ was optimal when considering image quality, dose, and reconstruction time. The future of $4 D C B C T$ clinical utility relies on further investigation of image acquisition and reconstruction optimization.
\end{abstract}

Keywords: Cone-beam CT, Image guidance, Dosimetry, Motion management

\footnotetext{
* Correspondence: nwen1@hfhs.org

${ }^{3}$ Department of Radiation Oncology, Henry Ford Health System, Detroit, MI 48202, USA

Full list of author information is available at the end of the article
} 


\section{Background}

Stereotactic body radiotherapy (SBRT) has become a form of treating inoperable non-small cell lung cancer (NSCLC) in its early stages [1]. Work conducted by Onishi et al. showed that patients staged with T1 and T2 NSCLC treated with SBRT had cumulative local control rates better than $70 \%$ at 5 years; this is in contrast to local control rates of $50 \%$ with a 5-year survival of approximately 15-30\% for patients treated with conventionally fractionated radiotherapy [1]. Five-year relative survival rates for lung cancers remains somewhat low at $18 \%$, attributed to more than one-half of diagnoses made at a distant stage [2].

Though initial clinical results of SBRT for lung cancer are promising, there remain technical complexities that must be addressed. In particular, localization error associated with the treatment of moving targets in lung SBRT must be minimized [3]. The ability of fourdimensional CT (4DCT) to map motion and tissue deformation during respiration, while reducing artifacts, allows for accurate targeting of tumors in the thorax $[4,5]$. Strategies to improve target coverage, such as breath hold treatments, gated delivery, and mid-position treatments can also be utilized as part of an approach for respiratory motion management $[4,6,7]$. Margin reduction is also possible for SBRT, having subsequent implications for reducing mean lung dose [8].

Interventional imaging techniques such as conebeam CT (CBCT) have become clinical standards in image-guided radiotherapy for soft tissue-based target localization and positioning [9]. Linear accelerators can be mounted with a $\mathrm{kV}$ source and flat panel detector, allowing for volumetric image acquisition. These volumetric images allow for accurate soft tissue localization and retrospective dose calculation [10]. However, large magnitudes of intra-scan motion in the reconstructed image can lead to clinically dosimetric discrepancies due to poor image quality.

Tumor motion at the treatment position can be assessed using 4DCBCT [11]. Conventional free-breathing CBCT tends to underestimate the tumor extent (i.e., the internal target volume) by as much as 24.2 to $40.1 \%$ depending on tumor size and interfraction variability [3, 12, 13]. To allow for binning of projection images, a surrogate respiratory signal is used to determine amplitude, phase, or temporal information; various methods have been utilized for the respiratory cycle surrogate, including tracking the motion of the diaphragm, mapping changes of the skin surface, thoracic transducer belts, or infrared reflective markers [14]. The 4D reconstruction involves a retrospective correlation of the timing of projection images to the breathing cycle surrogate parameter of interest (i.e., phase or amplitude of respiratory cycle) $[4,15]$.

The clinical utility of $4 \mathrm{DCBCT}$ is dependent on both image quality and imaging dose. Dosimetric studies have been conducted for patient skin dose using thermoluminescent dosimeters as well as Gafchromic film inserted into homogeneous phantoms for different CBCT protocols $[16,17]$. Wen et al. reported cumulative dose levels to the left femoral head from daily $\mathrm{kV}$ CBCT of pelvic sites can be upwards of $400 \mathrm{cGy}$. Since 4DCBCT has great potential in localizing lesions in the thorax, it would be worthwhile to understand the dose delivered from 4DCBCT in a heterogeneous medium and the effect of changing practical variables in 4D protocols such as gantry speed due to gantry speed's inverse relationship with the number of projections. This study investigates the effect of gantry speed on 4DCBCT image quality and dose using the On-Board Imager $^{\oplus}(\mathrm{OBI})$ on the Edge ${ }^{\mathrm{TM}}$ radiosurgery system (Varian Medical System, Palo Alto, CA).

\section{Materials and methods \\ CBCT parameterization}

All images were acquired with $125 \mathrm{kVp}$ x-ray tube setting. Phantoms were placed at isocenter and were imaged using a half-fan field of view with a half-bowtie filter and full trajectory slightly greater than $360^{\circ}$ acquisition, reflecting a typical thoracic region protocol in which a larger field of view is required to prevent truncation of anatomy (see Table 1). A relatively large focal spot of $1.0 \mathrm{~mm}$ was used to mediate heat loss. The source-to-detector distance was $150 \mathrm{~cm}$. These fixed parameters mirrored a standard thoracic CBCT clinical protocol.

The standard Feldkamp-Davis-Kress (FDK) reconstruction algorithm was used in producing average

Table 1 Technique factors and parameters set with a variable gantry speed. All images were acquired with $125 \mathrm{kVp}$ x-ray tube setting

\begin{tabular}{|c|c|c|c|c|c|c|c|c|}
\hline Gantry speed ( $\%$ s) & Field of view $(\mathrm{cm})$ & Matrix size & Pixel size (mm) & Slice thickness (mm) & Tube voltage (kVp) & $\mathrm{mAs}$ & Projections & $\Delta t(\min )$ \\
\hline 1.0 & 46.5 & $512 \times 512$ & 0.7 & 2.0 & 125 & 5716 & 5400 & 6.0 \\
\hline 2.0 & 46.5 & $512 \times 512$ & 0.7 & 2.0 & 125 & 2856 & 2700 & 3.0 \\
\hline 3.0 & 46.5 & $512 \times 512$ & 0.7 & 2.0 & 125 & 1903 & 1800 & 2.0 \\
\hline 4.0 & 46.5 & $512 \times 512$ & 0.7 & 2.0 & 125 & 1427 & 1350 & 1.5 \\
\hline 5.0 & 46.5 & $512 \times 512$ & 0.7 & 2.0 & 125 & 1140 & 1080 & 1.2 \\
\hline 6.0 & 46.5 & $512 \times 512$ & 0.7 & 2.0 & 125 & 949 & 900 & 1.0 \\
\hline
\end{tabular}


(CBCT-ave) and 4D reconstructions for all acquisitions [18]. Projections were sorted retrospectively into bins according to their respiratory phase corresponding to a surrogate signal, as seen in Fig. 1. The surrogate signal was generated by placing an infrared reflective marker block on a moving anterior-posterior (AP) platform of a BrainLAB ExacTrac gating system phantom (BrainLAB, Heimstetten, Germany) used in tandem with the realtime position management (RPM) system $^{\text {Th }}$ (Varian Medical Systems, Palo Alto, CA) to track the motion of the block (see Fig. 2) [19]. An independent computer controlled the motion of the gating phantom's platforms. A well-defined sinusoidal waveform simulated regular breathing cycles, representing an idealized model for tumor motion [20-22]. The amplitude of waveform motion $A$ in $\mathrm{cm}$ as a function of phase $\phi$ is given by:

$$
A=A_{o}(1-\cos (2 \pi \phi))^{2} ; \phi \in[0,1]
$$

where $A_{0}$ represents an amplitude constant in $\mathrm{cm}$. The RPM system ${ }^{\text {Tn }}$ tracks the block's motion with an infrared camera and assigns projections to appropriate phase bins used for retrospective reconstruction. The Catphan ${ }^{\circ} 504$ phantom was used to evaluate CBCT image quality (Phantom Laboratory, Salem, NY) by placing it on a lower moving superior-inferior (SI) platform of the gating phantom that moved in phase with the infrared marker block's platform.

An array of parameters can be varied for $\mathrm{CBCT}$ acquisition. The number of projections acquired directly relates to image quality and imaging dose [23]. The number of projections $N$ for a given $\mathrm{CBCT}$ protocol is determined by:

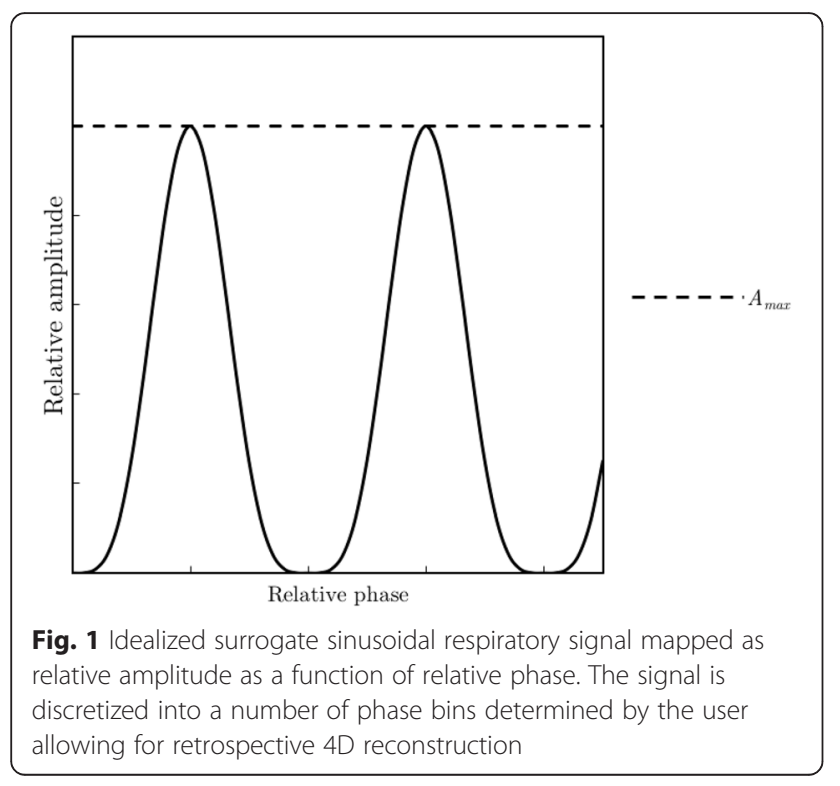

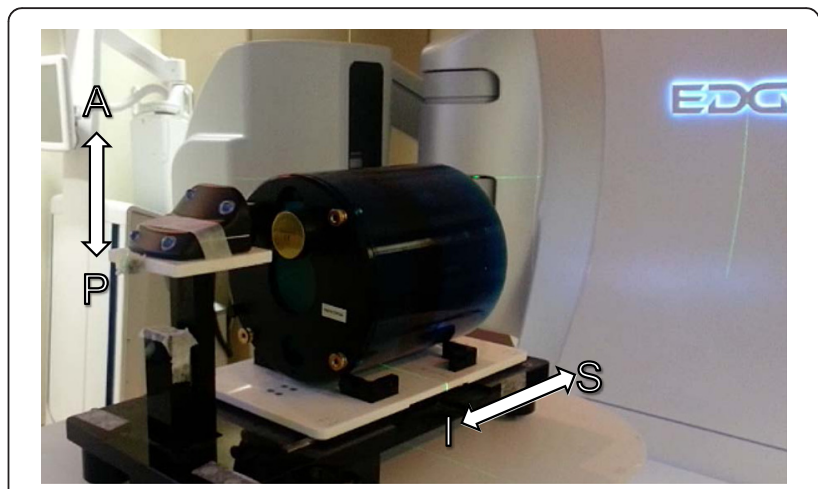

Fig. 2 Equipment used in $4 \mathrm{D}$ analysis. The Catphan sits on the SI platform while the block sits on the AP platform. The motion of the block is recorded and used as the surrogate signal in $4 \mathrm{D}$ reconstruction

$$
N=F \Delta t=F(\theta / \omega)
$$

where $F$ is the frame rate and $\Delta t$ is the acquisition time determined by the angular displacement $\theta$ and inversely by the gantry speed $\omega$. Thus, $N$ varies inversely with $\omega$. Six protocols were designed by varying gantry speed in integer steps from 1.0 to $6.0^{\circ}$ per second $(\% / \mathrm{s})$ at a fixed frame rate of 15 frames per second (fps) (see Table 1).

\section{Image quality analysis}

The following image quality parameters were evaluated: spatial resolution, low contrast detectability, uniformity, and difference in Hounsfield unit (HU) sensitivity from baseline. Image quality metrics were determined for CBCT-ave for all gantry speeds. Modules for image quality evaluation are shown in Fig. 3. All image quality measurements were performed using the Eclipse treatment planning system (Varian Medical Systems, Palo Alto, CA). Certain image quality parameters specified below were normalized to the square root of the $\mathrm{mAs}$, as this represents the anticipated relative noise of the image and provides a kind of benefit-cost ratio as it pertains to image quality.

\section{Spatial resolution}

A bar pattern module (CTP528) was used to determine the modulation transfer function (MTF) of every protocol to completely characterize the spatial resolution of the imaging system, as seen in Fig. 3a. The spatial frequency $f$ of the bar patterns are well known. By calculating the modulation of the bar patterns for various frequencies [24], a raw MTF can be generated via:

$$
M T F_{\text {raw }}(f)=\frac{H U_{\max }(f)-H U_{\min }(f)}{H U_{\max }(f)+H U_{\min }(f)}
$$

These metrics were determined by taking line intensity profiles across the bar patterns. Gaussian fits were applied 


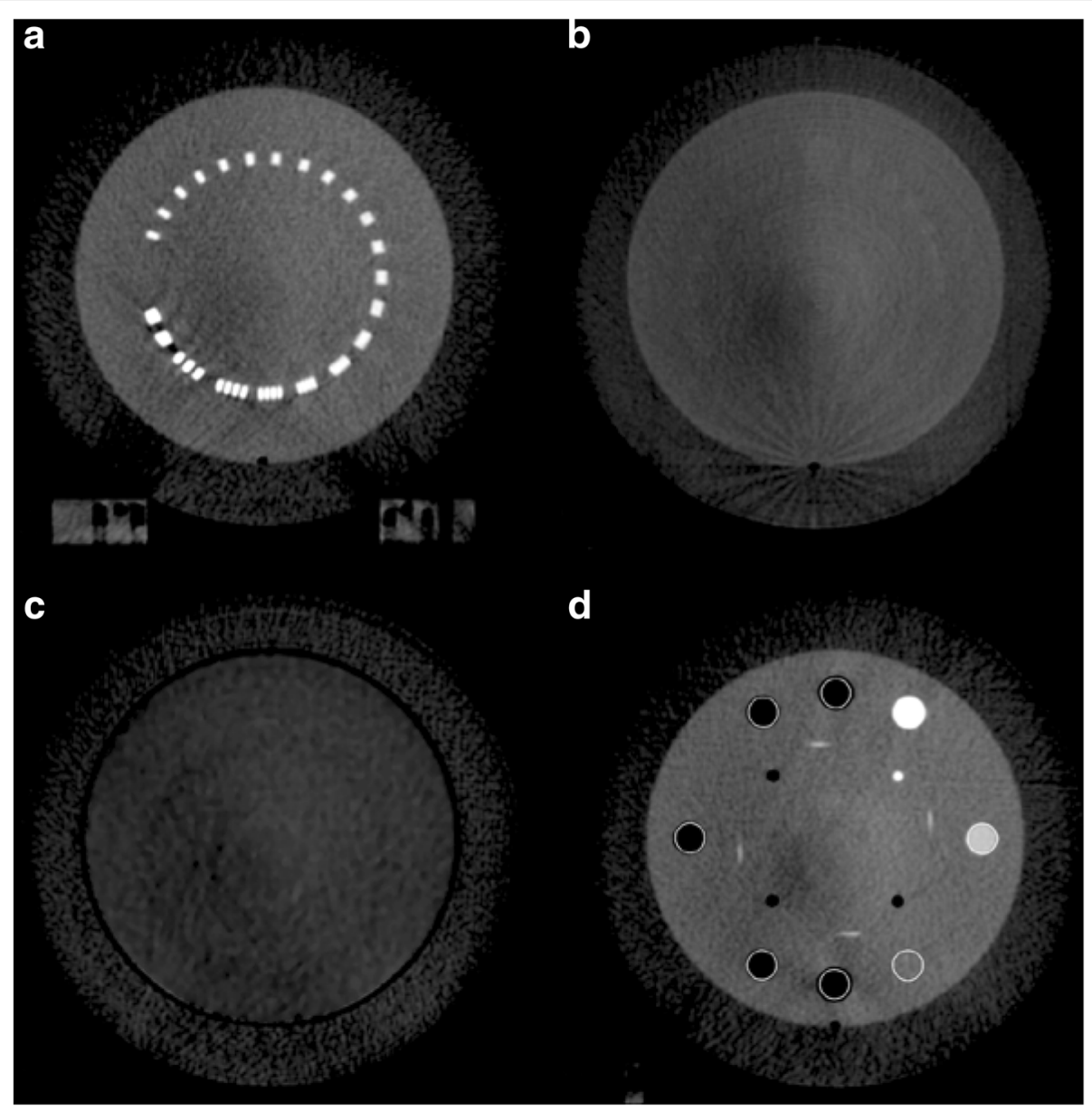

Fig. 3 Different slices of the Catphan's modules used for image quality evaluation. a A bar pattern module of increasing spatial frequency (Ip/ $\mathrm{cm})$, used for determining spatial resolution of the protocols. $\mathbf{b}$ A low contrast module used in calculating CNR of the various protocols. $\mathbf{c} A$ homogeneous module used in determining uniformity, with four ROI (anterior, posterior, right, and left) equidistant from a centrally located ROI. d A sensitometry module containing materials of known electron density, used in the calculating HU difference from baseline

to the raw data by maximizing the coefficient of determination $\left(R^{2}\right)$. The final MTF is given by:

$$
\operatorname{MTF}(f)=\exp \left(-f^{2} / 2 \sigma^{2}\right)
$$

where $\sigma$ represents the fitting parameter. The maximal spatial resolution $f_{\max }$ is defined as the frequency at which the MTF crosses the $10 \%$ level. This was assessed for every protocol using the Gaussian fits. Variation of $f_{\max }$ normalized to the square root of mAs was also evaluated with respect to mAs.

\section{Low contrast detectability}

Quantification of low contrast detectability can be accomplished via calculation of the contrast-to-noise ratio (CNR). A module containing low contrast cylinders (CTP515) was used to determine CNR, as seen in Fig. 3b. A $1.5 \mathrm{~cm}$ in diameter, $1.0 \%$ nominal low contrast cylinder was defined as the region-of-interest (ROI). The CNR is defined as:

$$
C N R=\frac{\left|\bar{x}_{s}-\bar{x}_{b g}\right|}{\overline{\sigma_{b g}}}
$$

where $\bar{x}_{s}$ represents the mean $\mathrm{HU}$ in the ROI, $\bar{x}_{b g}$ represents the mean $\mathrm{HU}$ of the adjacent background, and $\sigma_{b g}$ represents the standard deviation of the background. CNR was calculated for ten individual slices and then averaged producing a single CNR for every protocol. Variation of CNR normalized to the square root of $\mathrm{mAs}$ was also evaluated with respect to mAs.

\section{Image uniformity}

A module composed of homogeneous material (CTP486) was used to determine image uniformity. The mean $\mathrm{HU}$ values of four ROI $5.0 \mathrm{~cm}$ equidistant 


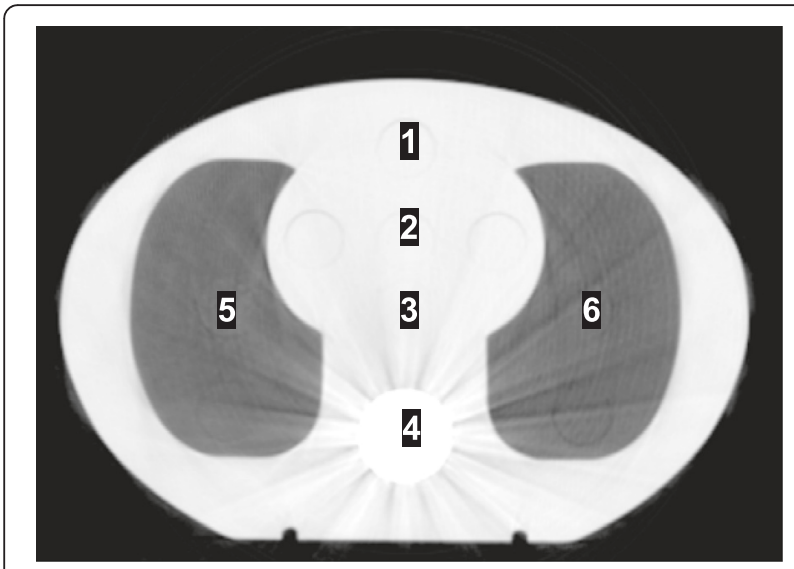

Fig. 4 lon chamber measurement points within the CIRS IMRT Thorax phantom

from a centrally located ROI were determined, as seen in Fig. 3c. The uniformity index (UI) is given by:

$$
U I=H U_{\max }-H U_{\min }
$$

UI was calculated for ten individual slices and then averaged producing a single UI for every protocol. Variation of UI normalized to the square root of mAs was also evaluated with respect to mAs.

\section{HU sensitivity}

An imaging system's ability to accurately characterize a given material's electron density (ED) is the essence of HU sensitivity. This was accomplished via measurement of various cylinders found in the Catphan's sensitometry module (CTP404), as seen in Fig. 3d. The mean HU value of each cylinder was measured over ten individual slices and then averaged producing a single mean $\mathrm{HU}$ for each cylinder for every protocol. The difference in mean $\mathrm{HU}$ from a baseline CBCT scan without motion performed at the same $\mathrm{kVp}$ was calculated for every cylinder. Ground truth of a given material's $\mathrm{HU}$ value is represented by the baseline scan [25]. This was used to construct a curve for quantification of $\mathrm{HU}$ sensitivity.

\section{D analysis}

Retrospective 4D reconstructions were performed maintaining the volume-of-interest while varying reconstruction with 4 uncorrelated phases (CBCT-4 ph) or 10 uncorrelated phases (CBCT-10 ph) as a means to measure differences in reconstruction quality. Reconstruction time increases linearly with the number of projections as well as the number of phases (e.g. approximately 6 and $20 \mathrm{~min}$ for CBCT-4 ph and CBCT-10 ph, respectively with 5400 projections). Excursion of this study was $3.0 \mathrm{~cm}$ in the SI direction and was calculated by taking the difference in the maximum and minimum position of the air cylinder sensitometry insert. Percent differences between expected excursion and those measured on the $4 \mathrm{DCBCT}$ were calculated. To compare excursion between CBCT-4 ph and CBCT-10 ph, root-mean square error (RMSE) for excursion was determined across the different protocols, as projection data for each protocol was independently acquired. For some measured excursion $E$ for a given protocol $i$, the RMSE for a given $4 \mathrm{D}$ reconstruction is given by:
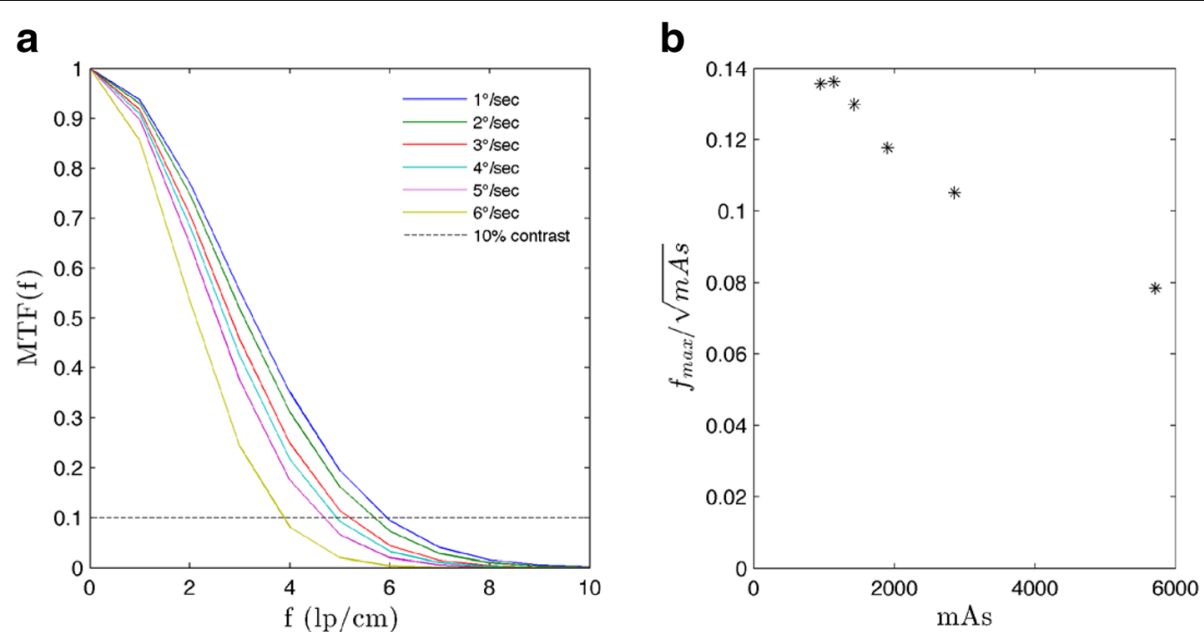

Fig. 5 a Gaussian fit MTF for CBCT-ave for the various protocols. b Maximal spatial resolution normalized to the square root of $m A$ s. As defined above, the maximal spatial resolution corresponds to the spatial frequency at which the MTF crosses the $10 \%$ contrast level 
Table 2 Maximal spatial resolution $(\mathrm{lp} / \mathrm{cm})$ for CBCT-ave at specified gantry speeds

\begin{tabular}{llllll}
\hline \multicolumn{6}{l}{ Gantry speed $(\% / s)$} \\
\hline 1.0 & 2.0 & 3.0 & 4.0 & 5.0 & 6.0 \\
5.93 & 5.62 & 5.14 & 4.91 & 4.60 & 3.82 \\
\hline
\end{tabular}

These spatial frequencies correspond to $10 \%$ contrast of the Gaussian fit

$$
R M S E=\sqrt{\frac{1}{6} \sum_{i=1}^{6}\left(E_{i}-3.0\right)^{2}}
$$

This was done for both $4 \mathrm{D}$ reconstruction techniques.

\section{Reference dosimetry}

Following the recommendations of AAPM Task Group 61, an IBA CC13 ionization chamber (IBA Dosimetry $\mathrm{GmbH}$, Schwarzenbruck, Germany), was used to provide absolute dose information [26]. The CC13 was calibrated at an Accredited Dosimetry Calibration Laboratory with a tungsten target, aluminum filtration beam in the $\mathrm{kV}$ energy range at 100 and $120 \mathrm{kVp}$, with reported halfvalue layers (HVL) of 5.0 and $6.8 \mathrm{~mm} \mathrm{Al}$ respectively. The HVL for the $125 \mathrm{kVp}$ spectrum of the OBI was determined in air using Gammex high purity aluminum attenuators (Gammex, Middleton, WI). Doses were measured with an electrometer (Keithley 35040 SN 70422) by placing the chamber (IBA CC13 SN 7406) at different coplanar locations within a CIRS IMRT thorax phantom (Model 002LFC, CIRS Inc, Norfolk, VA) placed upon the gating phantom and using the same waveform motion as the Catphan. The plane resided in the central axis at rest. The CIRS is manufactured using proprietary materials intended to mimic the attenuation characteristics of water, bone, and lung within $3.0 \%$ for energies ranging from $50 \mathrm{keV}$ to $25 \mathrm{MeV}$. The chamber was placed in regions of the CIRS phantom described in Fig. 4a (regions 1-3 for water, region 4 for a bone, and regions 5 and 6 for lung) for every protocol. These measurements were used to determine dose rates in the different tissue mimicking materials.

\section{Results}

\section{A. Image quality evaluation}

Spatial resolution was evaluated for CBCT-ave. The spatial resolution data are shown in Fig. 5a, exhibiting linearly decreasing behavior with increasing gantry speed. Linearly decreasing maximal spatial resolution with increasing gantry speed is exhibited in Table 2 . Evaluation of maximal spatial resolution normalized to the square root of $\mathrm{mAs}$ a maximum value at $1140 \mathrm{mAs}$ as seen in Fig. 5b. The inverse square root term in the $f_{\text {max }}$ normalization dominates as $\mathrm{mAs}$ increases.

Low contrast detectability exhibited linear decreases, with CNR variations from 4.80 to 1.82 as gantry speed increased from 1.0 to $6.0^{\circ} \mathrm{s}$ as seen in Fig. 6a. Evaluation of CNR normalized to the square root of $\mathrm{mAs}$ indicates small increases up to $1427 \mathrm{mAs}$ as seen in Fig. 6b. The inverse square root term in the CNR normalization dominates as mAs increases. Qualitative variation of low contrast detectability is exhibited in Fig. 7.

Minimal variations between protocols were observed for image uniformity, as seen in Fig. 8. The difference between the maximum and minimum UI across all the
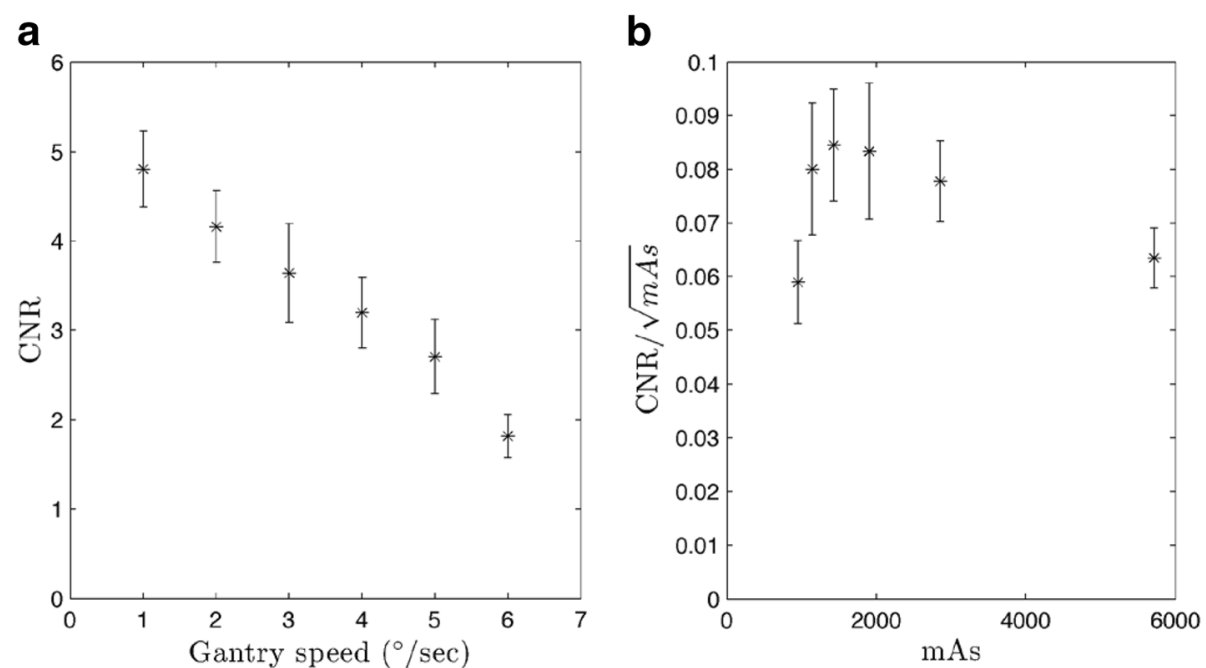

Fig. 6 CNR for CBCT-ave. All values were calculated over ten individual slices and then averaged. Error bars represent standard error. a CNR exhibits a linear trend as a function of gantry speed. $\left(R^{2}=0.988\right)$. $\mathbf{b} C N R$ normalized to the square root of mAs. Values were calculated over ten individual slices and then averaged. All error bars represent standard error 


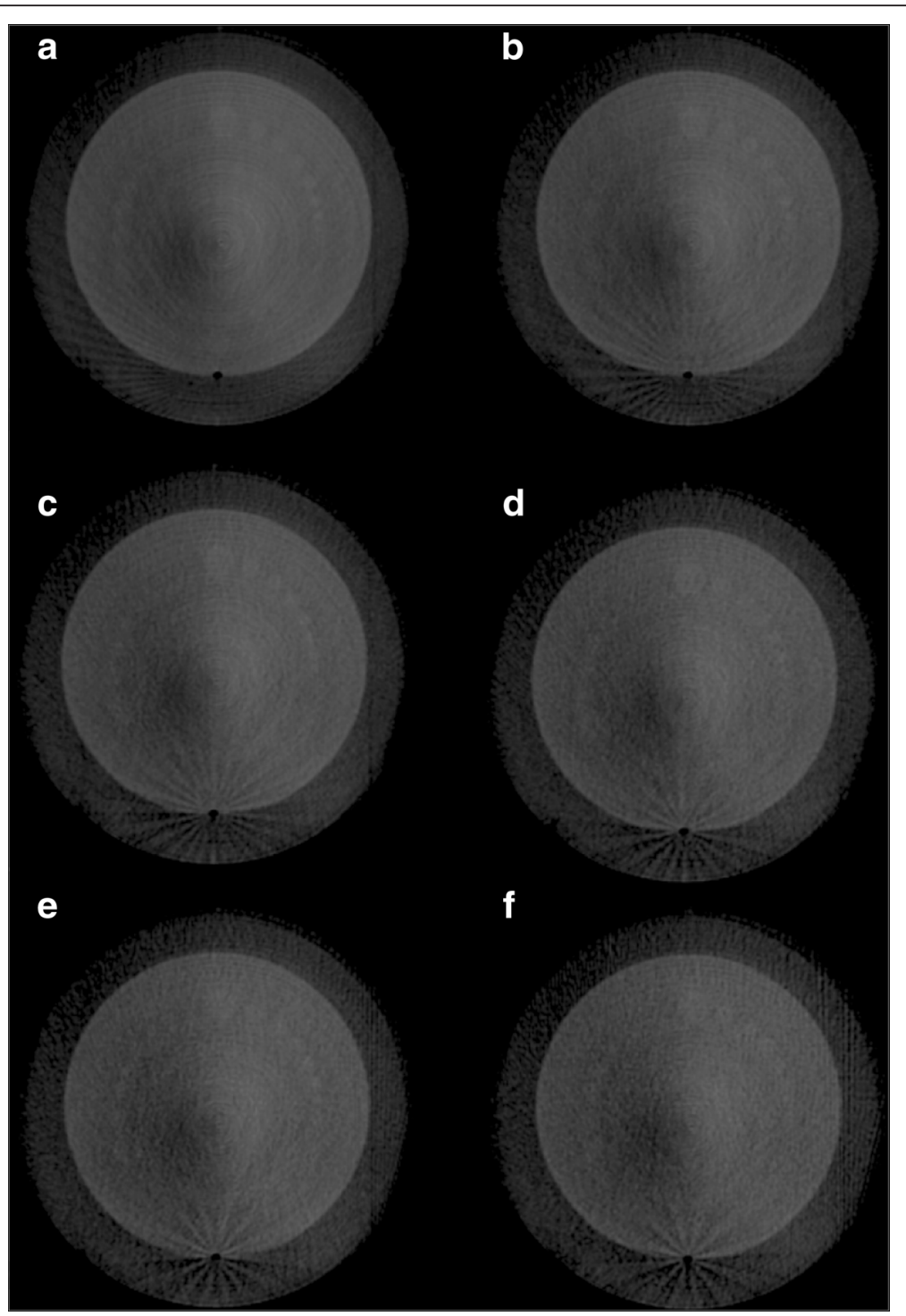

Fig. 7 Low contrast module of the Catphan. Images correspond to gantry speeds varying from 1.0 to $6.0 \%$ for (a) through (f) respectively

protocols is less than $3.0 \mathrm{HU}$, as seen in Fig. 8a. Evaluation of UI normalized to the square root of $\mathrm{mAs}$ indicates complete dominance of the inverse square root term as seen in Fig. 8b.

Minimal variations were also observed for HU sensitivity differences from baseline, as seen in Fig. 9. The largest variation in mean $\mathrm{HU}$ between protocols was observed for the air cylinder of $\mathrm{ED}=0$. The difference between the maximum and minimum average $\mathrm{HU}$ for air was $100 \mathrm{HU}$. However, all average $\mathrm{HU}$ values are within one standard deviation of each other, in turn making the HU-to-ED curves even more indistinguishable.

Variations in excursion across 4D reconstruction techniques for all protocols were also minimal. The difference between the maximum and minimum measured excursion across all protocols and all reconstruction techniques was less than $1.0 \mathrm{~mm}$. The RMSE in excursion for CBCT-4 ph and CBCT-10 ph were 0.025 and $0.035 \mathrm{~cm}$, respectively. Percent differences in excursion are presented in Table 3. No relationship in error to the known $3.0 \mathrm{~cm}$ excursion with the number of projections was observed.

\section{Imaging dose evaluation}

The HVL measured in air was determined to be $5.02 \mathrm{~mm} \mathrm{Al}$. Appropriate mass-energy absorption coefficients were applied to the tissue-mimicking materials [26] Resultant dose rates for a given chamber position are provided in Table 4 below. Comparison 

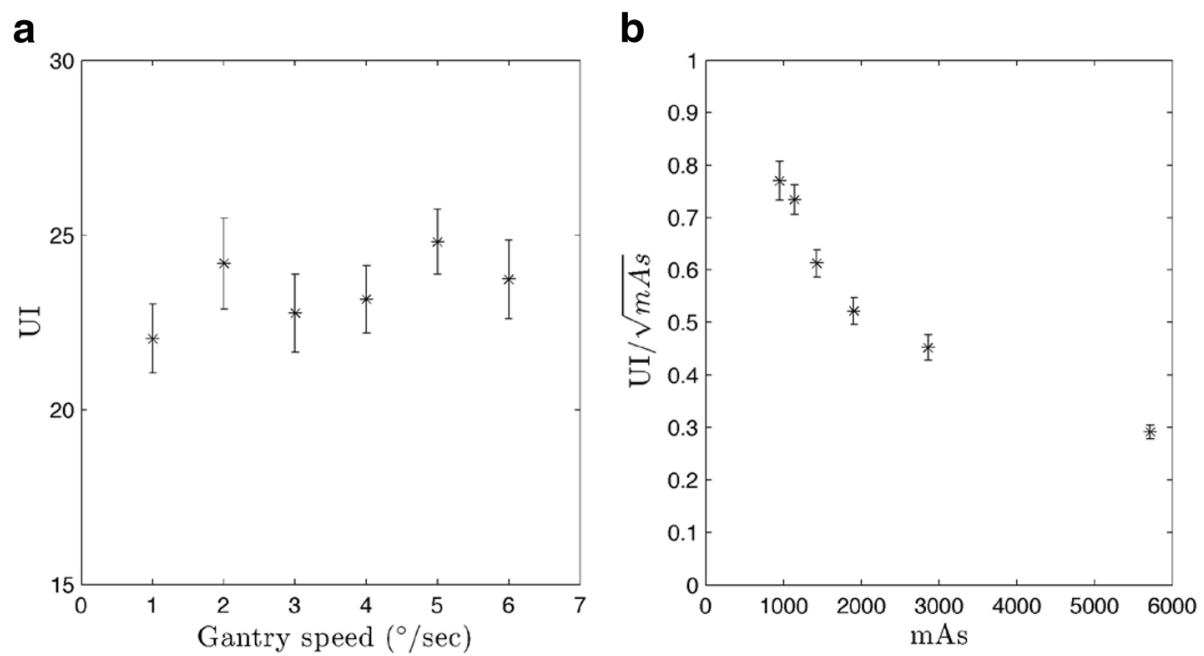

Fig. $8 \mathrm{UI}$ for CBCT-ave plotted against a gantry speed and $\mathbf{b}$ normalized to mAs. Values were calculated over ten individual slices and then averaged. All error bars represent standard error

to other dosimetry studies is provided in the discussion below.

\section{Discussion}

$\mathrm{CBCT}$ finds great utility in radiation oncology. $\mathrm{CBCT}$ image acquisition prior to delivery of radiation can be registered to previously acquired CT scans used for treatment planning and provides images for retrospective dose accumulation studies. However, standard 3D acquisition and reconstruction does not allow visualization of mobile lesions as a function of respiratory phase and is susceptible to motion artifacts such as blurring and distortion. Moreover, average 3D reconstructions may result in underestimates in excursion due to short periods of time spent at peak inhalation/exhalation. The use of 4DCBCT has the potential to mitigate these issues by assigning

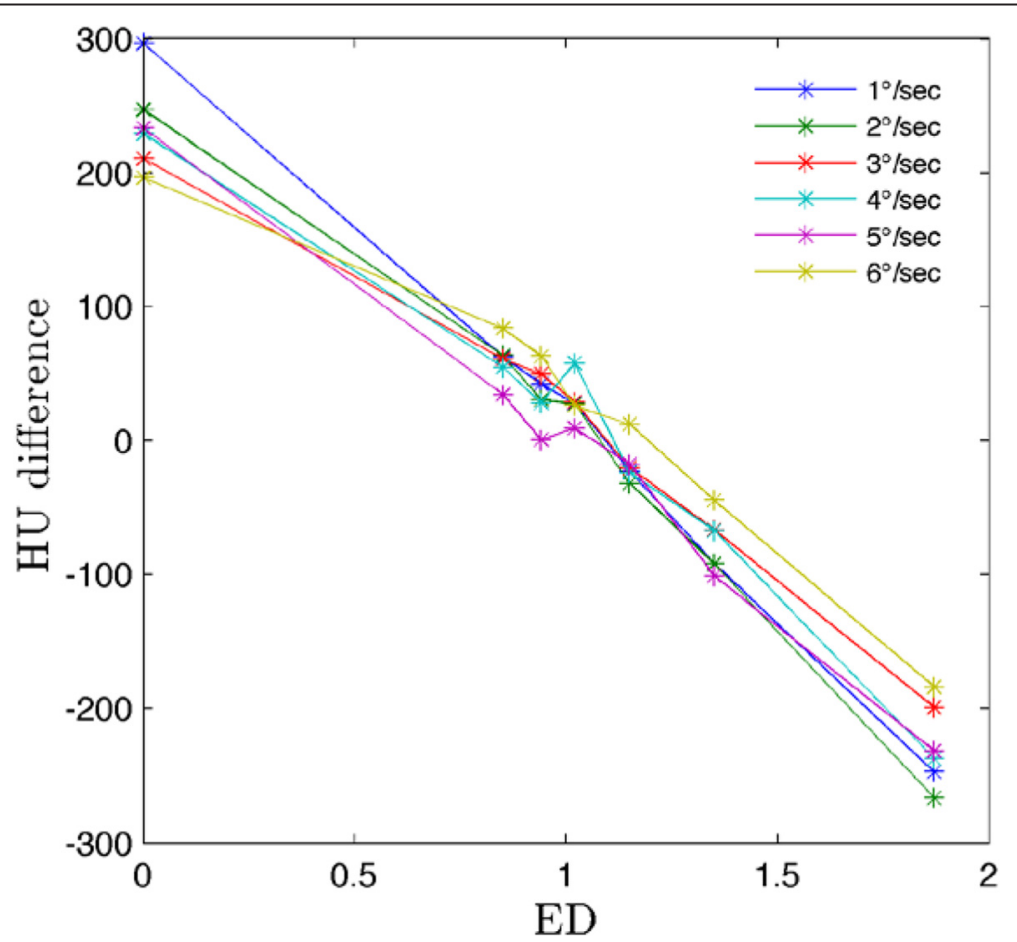

Fig. 9 Difference in mean HU from baseline for CBCT-ave. Values were measured over ten individual slices and then averaged 
Table 3 Percent differences in excursion for the various protocols

\begin{tabular}{lrlllllll}
\hline & \multicolumn{9}{c}{ Gantry speed $(\% / \mathrm{s})$} & & & \\
\cline { 2 - 7 } Volume & 1.0 & 2.0 & 3.0 & 4.0 & 5.0 & 6.0 & RMSE (cm) \\
\hline CBCT-4 ph & -1.34 & 1.32 & -1.34 & 0.00 & -1.68 & 0.00 & 0.025 \\
CBCT-10 ph & 1.32 & 1.00 & -0.33 & 0.00 & 1.00 & 0.33 & 0.035 \\
\hline
\end{tabular}

Positive values represent overestimates while negative values represent underestimates. RMSE across the protocols for a given reconstructed volume is also provided

projections to specific breathing cycle phases. This is feasible due to changes in the diaphragm, causing organ motion with the diaphragm's dynamics. This in turn justifies the use of surrogate signals such as skin surface mapping or infrared markers, as the position of the surrogate is variable with respect to time [4, 7].

A major difficulty in $4 \mathrm{DCBCT}$ is the limited number of projections per phase bin. Insufficient projection data leads to aliasing artifacts. $\mathrm{Li}$ and Xing proposed slow gantry rotation (SGR) and multiple gantry rotation (MGR) acquisition techniques to increase the number of projections for a given phase [27]. They showed SGR produced superior images to MGR for the same mAs when compared to 3DCBCT counterparts.

Time-averaged 4DCBCT images over the respiratory cycle are used for patient localization compared against time-averaged 4DCT images [28]. The image quality of $4 \mathrm{DCBCT}$ images plays an important role in 4D dose accumulation. Anatomical voxels are mapped from the $4 \mathrm{DCBCT}$ at treatment position to a reference $4 \mathrm{DCT}$. However, the low number of projections per phase in the $4 \mathrm{DCBCT}$ image may result in degraded image quality, specifically streaking at high contrast boundaries and blurring, in turn leading to unrealistic dose calculations [29].

In this study, contrast-based image quality metrics were affected by variable gantry speed. Yoganathan et al. have shown similar results in their analysis of an Elekta 4DCBCT system using a Catphan ${ }^{\circledR} 600$ [30]. Specifically, spatial resolution and low contrast detectability decreased with increasing gantry speed and a fixed frame rate. Yoganathan et al. further demonstrated that reductions in $4 \mathrm{DCBCT}$ image quality parameters may result in underestimates of target volumes when compared to 4DCT contoured volumes used in treatment planning.

Table 4 Dose rates (E-3 cGy/mAs) measured in different tissuemimicking materials

\begin{tabular}{llllll}
\hline \multicolumn{6}{l}{ Chamber position } \\
\hline 1 & 2 & 3 & 4 & 5 & 6 \\
2.86 & 3.03 & 2.84 & 5.18 & 2.49 & 2.30 \\
\hline
\end{tabular}

Improved metrics with slower acquisition leading to increased projections/mAs is likely due to decreases in noise. Saturation of these metrics occurs when viewing the parameters against the number of projections/mAs. This is related to the imaging system being unable to decrease noise levels further. However, normalization of image quality parameters by the square root of mAs indicates marginal improvements in maximal spatial resolution and low contrast detectability passed a given mAs and a lack of improvement with respect to uniformity. The presence of artifacts like streaking, as seen in Fig. 10, makes evaluation of image quality for 4D volumes difficult. Cooper et al. have described streaking and star artifacts in $4 \mathrm{DCBCT}$ being attributed to the presence of two sampling frequencies - a higher sampling frequency for the projections in a given phase bin and a lower sampling frequency between phase bins [31]. In implementing a FDK cone-beam reconstruction with so few projections per phase, materials with stronger attenuation characteristics are not balanced out in the back projection process in turn leading to star and streaking artifacts [18].

HU sensitivity and excursion did not exhibit noteworthy differences in CBCT-ave with variable gantry speed, especially when accounting for error in the case HU sensitivity. With respect to excursion, across all the protocols, percent differences were less than $2.0 \%$. In comparing 4 phase against 10 phase reconstructions, both RMSE are less than half a millimeter. This indicates that the 4D reconstructions reproduce realistic excursion. In this particular study, the $15 \mathrm{fps}$ acquisition and characteristics of the waveform motion mitigate issues of latency. Based on the motion waveform used, acquisition may miss $0.8 \mathrm{~mm}$ of motion between frames on average. For more irregular breathing patterns, this may not be the case resulting in errors in the phase sorting process.

Characterization of image quality for given tube current settings (mAs) for 4DCBCT has been previously performed for systems including the Varian's Acuity ${ }^{\text {TM }}$ simulator and Trilogy ${ }^{\mathrm{Tm}}$ OBI (Varian Medical Systems, Palo Alto, CA) $[25,29]$. These studies focused primarily on a comparison of image quality relative to some reference using SGR or MGR acquisition techniques. Dose was characterized via mAs, as dose is directly proportional to mAs [29].

The $\mathrm{CTDI}_{100}$ metric is not appropriate for dosimetric evaluation of CBCT scans due to its inability to accommodate and record the whole primary beam and scattered radiation [32]. Hence 4DCBCT was evaluated dosimetrically on an absolute scale. Ion chamber measurements from this study produced dose rates of $3.0 \times$ $10^{-03} \mathrm{cGy} / \mathrm{mAs}$ at isocenter in a water equivalent medium. Li et al. reported for the Varian Acuity and Trilogy systems, a $125 \mathrm{kVp}$ spectrum and tube current 


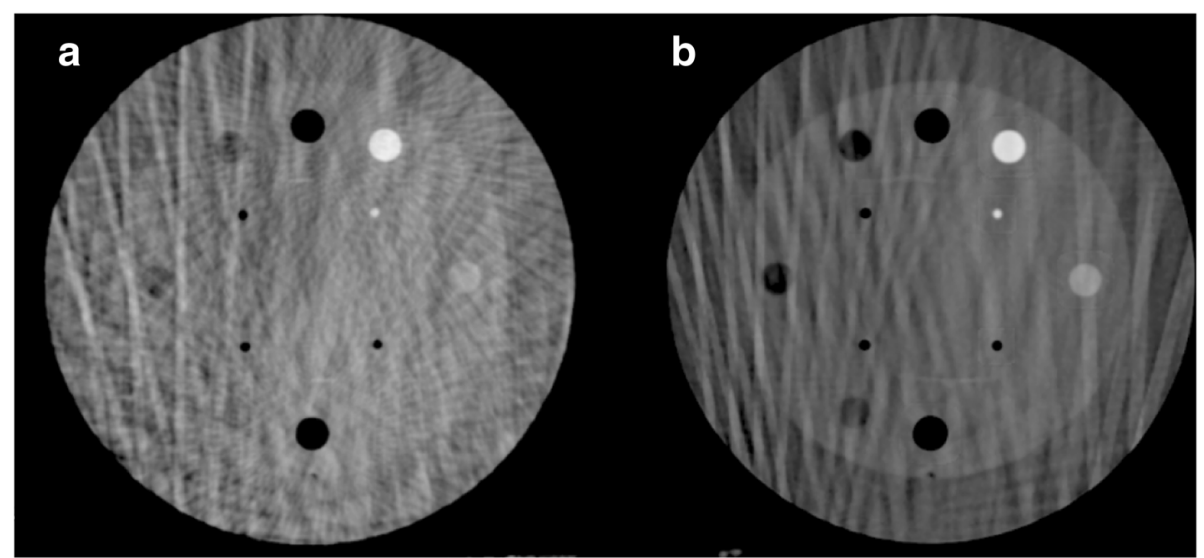

Fig. 10 Streaking artifacts present in the sensitometry module for the same CBCT slice and the same phase. These slices correspond to a CBCT4 ph and $\mathbf{b}$ CBCT-10 ph for a gantry speed of $1 \% \mathrm{~s}$

of $80 \mathrm{~mA}$ produced dose rates at isocenter of $2.92 \times 10$ -03 and $2.79 \times 10^{-03} \mathrm{cGy} / \mathrm{mAs}$, respectively [33]. Similarly, Gardner et al. reported isocenter doses using film in a Wellhofer phantom of $(2.72 \pm 0.11) \times 10^{-03}$ cGy/ $\mathrm{mAs}$ using TrueBeam OBI and $125 \mathrm{kVp}$ setting. McMillan et al. compared measured dose rates to dose rates determined using Monte Carlo methods for the OBI kV imaging system integrated into the Varian Novalis Tx radiosurgery platform (Varian Medical Systems, Palo Alto, CA) [34]. A dose rate of $2.23 \times 10^{-03} \mathrm{cGy} / \mathrm{mAs}$ at isocenter was calculated for a comparable pelvic $\mathrm{CBCT}$ protocol using a $125 \mathrm{kVp}$ spectrum and a $32 \mathrm{~cm}$ diameter cylindrical CTDI phantom composed of homogeneous polymethyl methacrylate. Variation in dose rate in this study from others is attributed to spectral differences and variations in phantom composition and geometry.

While increasing the cumulative $\mathrm{mAs}$ increases the quality of spatial resolution and low contrast detectability, the cost of linearly increasing dose and increased 4D image acquisition and reconstruction time must be considered. Artifacts such as streaking in $4 \mathrm{DCBCT}$ are largely attributed to the lack of projection data being used for reconstruction. In this particular study, the incremental improvements in image quality above 2000 projections become unjustified compared to the linear increases in dose. This corresponds to a gantry speed between 2 and $3 \%$ s. Clinical use of 4DCBCT systems for patients relies entirely on intended application.

\section{Conclusions}

A quantitative analysis of the Varian OBI's 4DCBCT capabilities was explored for making clinical decisions. The effects of gantry speed on 4DCBCT image quality and dose have been investigated yielding anticipated results. When using the same technique settings $(\mathrm{kVp})$, variations in gantry speed change the number of projections used for reconstruction but maintain the mAs per projection. Unsurprisingly, contrast-based image quality metrics were found to decrease linearly with increasing gantry speed but show marginal improvements when appropriately normalized to the square root of $\mathrm{mAs}$. The benefit of increased contrast comes at the cost of increased dose, slower acquisition time, and longer 4D reconstruction time; the clinical benefits of improved image quality must be weighed against the costs of decreased efficiency and increased imaging dose. The future of 4DCBCT's clinical utility relies on further investigation of image optimization, requiring more than just largescale increases in $\mathrm{mAs}$ to improve image quality.

\section{Abbreviations}

$4 D C T$, four-dimensional $C T$; $A P$, anterior-posterior; $C B C T$, cone-beam $C T$; CBCT-10 ph, ten phase $4 D C B C T$ reconstruction; $C B C T-4$ ph, four phase $4 D C B C T$ reconstruction; $C B C T$-ave, average $C B C T$ reconstruction; $C N R$, contrast-to-noise ratio; ED, electron density; FDK, Feldkamp-Davis-Kress; fps, frames per second; HU, Hounsfield unit; HVL, half-value layer; MGR, multiple gantry rotation; MTF, modulation transfer function; NSCLC, non-small cell lung cancer; OBI, On-Board Imager ${ }^{\oplus}$; RMSE, root-mean square error; ROI, region-of-interest; RPM, real-time position management; SBRT, stereotactic body radiotherapy; SGR, single gantry rotation; SI, superior-inferior; UI, uniformity index

\section{Acknowledgements}

The authors thank American Cancer Society for funding this project. The assistance from Richard Cattaneo II, MD, Radiation Oncologist at St. Joseph Mercy Hospital is especially appreciated.

\section{Funding}

This work was supported by a research scholar grant (RSG-15-137-01-CCE) from the American Cancer Society.

Availability of data and materials

All data and materials in the manuscript are freely available to any scientist.

Authors' contributions

APS, KHS, SJG, and NW designed the project. APS, KHS, and YQ contributed to acquisition of data. APS, KHS, SJG, and CL performed data analysis. All authors read and approved the final manuscript. 


\section{Competing interest}

The authors declare that they have no competing interests.

\section{Consent for publication}

Not applicable.

\section{Ethics approval and consent to participate}

Not applicable.

\section{Author details}

'Department of Radiation Oncology, Wayne State University School of Medicine, Detroit, MI 48201, USA. ${ }^{2}$ Texas Oncology, Fort Worth, TX 76104, USA. ${ }^{3}$ Department of Radiation Oncology, Henry Ford Health System, Detroit, Ml 48202, USA.

Received: 9 January 2016 Accepted: 22 July 2016

\section{Published online: 29 July 2016}

\section{References}

1. Onishi H, Shirato H, Nagata Y, Hiraoka M, Fujino M, Gomi K, et al. Stereotactic body radiotherapy (SBRT) for operable stage I non-small-cell lung cancer: Can SBRT be comparable to surgery? Int J Radiat Oncol Biol Phys. 2011;81:1352-8.

2. Siegel RL, Miller KD, Jemal A. Cancer statistics, 2015. CA Cancer J Clin. 2015; 65:5-29.

3. Vergalasova I, Maurer J, Yin FF. Potential underestimation of the internal target volume (ITV) from free-breathing CBCT. Med Phys. 2011;38:4689-99.

4. Colgan R, McClelland J, McQuaid D, Evans PM, Hawkes D, Brock J, et al. Planning lung radiotherapy using $4 \mathrm{D} C \mathrm{CT}$ data and a motion model. Phys Med Biol. 2008:53:5815-30.

5. Kruis MF, van de Kamer JB, Belderbos JS, Sonke JJ, van Herk M. 4D CT amplitude binning for the generation of a time-averaged 3D mid-position CT scan. Phys Med Biol. 2014:59:5517-29.

6. Peng Y, Vedam S, Chang JY, Gao S, Sadagopan R, Bues M, et al. 2011 Implementation of feedback-guided voluntary breath-hold gating for cone beam CT-based stereotactic body radiotherapy. Int J Radiat Oncol Biol Phys. 2011:80:909-17.

7. Wolthaus JW, Sonke JJ, van Herk M, Belderbos JS, Rossi MM, Lebesque JV, et al. Comparison of different strategies to use four-dimensional computed tomography in treatment planning for lung cancer patients. Int J Radiat Oncol Biol Phys. 2008;70:1229-38

8. Hof H, Rhein B, Haering P, Kopp-Schneider A, Debus J, Herfarth K. 4D-CTbased target volume definition in stereotactic radiotherapy of lung tumours: Comparison with a conventional technique using individual margins. Radiother Oncol. 2009;93:419-23.

9. Hioki K, Araki F, Ohno T, Nakaguchi Y, Tomiyama Y. Absorbed dose measurements for kV-cone beam computed tomography in image-guided radiation therapy. Phys Med Biol. 2014:59:7297-313.

10. Xing L, Thorndyke B, Schreibmann E, Yang Y, Li TF, Kim GY, et al. Overview of image-guided radiation therapy. Med Dosim. 2006;31:91-112.

11. Bergner F, Berkus T, Oelhafen M, Kunz P, Pa T, Grimmer R, et al. An investigation of $4 \mathrm{D}$ cone-beam $\mathrm{CT}$ algorithms for slowly rotating scanners. Med Phys. 2010;37:5044-53.

12. Ahmad M, Balter P, Pan T. Four-dimensional volume-of-interest reconstruction for cone-beam computed tomography-guided radiation therapy. Med Phys. 2011;38:5646-56.

13. Sonke JJ, Lebesque J, van Herk M. Variability of four-dimensional computed tomography patient models. Int J Radiat Oncol Biol Phys. 2008;70:590-8.

14. Park JC, Park SH, Kim JH, Yoon SM, Kim SS, Kim JS, et al. Four-dimensional cone-beam computed tomography and digital tomosynthesis reconstructions using respiratory signals extracted from transcutaneously inserted metal markers for liver SBRT. Med Phys. 2011;38:1028-36.

15. Leng S, Tang J, Zambelli J, Nett B, Tolakanahalli R, Chen GH. High temporal resolution and streak-free four-dimensional cone-beam computed tomography. Phys Med Biol. 2008;53:5653-73.

16. Gardner SJ, Studenski MT, Giaddui T, Cui Y, Galvin J, Yu Y, et al. Investigation into image quality and dose for different patient geometries with multiple cone-beam CT systems. Med Phys. 2008;41:031908.

17. Wen N, Guan H, Hammoud R, Pradhan D, Nurushev T, Li S, et al. Dose delivered from Varian's CBCT to patients receiving IMRT for prostate cancer. Phys Med Biol. 2007;52:2267-76.
18. Feldkamp L, Davis $L$, Kress J. Practical cone-beam algorithm. J Opt Soc Am A. 1984;1:612-9.

19. Chang Z, Liu T, Cai J, Chen Q, Wang Z, Yin FF. Evaluation of integrated respiratory gating systems on a Novalis Tx system. J Appl Clin Med Phys. 2011;12:3495.

20. Dietrich L, Jetter S, Tucking T, Nill S, Oelfke U. Linac-integrated 4D cone beam CT: first experimental results. Phys Med Biol. 2006;51:2939-52.

21. Lujan AE, Larsen EW, Balter JM, Ten Haken RK. A method for incorporating organ motion due to breathing into 3D dose calculations. Med Phys. 1999; 26:715-20.

22. Zhuang L, Yan D, Liang J, lonascu D, Mangona V, Yang K, et al. Evaluation of image guided motion management methods in lung cancer radiotherapy. Med Phys. 2014;41:031911.

23. Yan $\mathrm{H}$, Cervino L, Jia X, Jiang SB. A comprehensive study on the relationship between the image quality and imaging dose in low-dose cone beam CT. Phys Med Biol. 2012;57:2063-80.

24. Coltman J. The specification of imaging properties by response to a sine wave input. J Opt Soc Am. 1954;44:468-71.

25. Wen N, Glide-Hurst C, Nurushev T, Xing L, Kim J, Zhong H, et al. Evaluation of the deformation and corresponding dosimetric implications in prostate cancer treatment. Phys Med Biol. 2012;57:5361-79.

26. Ma CM, Coffey CW, DeWerd LA, Liu C, Nath R, Seltzer SM, et al. AAPM protocol for 40-300 kV x-ray beam dosimetry in radiotherapy and radiobiology. Med Phys. 2001;28:868-93.

27. Li T, Xing L. Optimizing $4 \mathrm{D}$ cone-beam $C T$ acquisition protocol for external beam radiotherapy. Int J Radiat Oncol Biol Phys. 2007;67:1211-9.

28. Rosu M, Hugo GD. Advances in $4 D$ radiation therapy for managing respiration: Part II - 4D treatment planning. Z Med Phys. 2012;22:272-80.

29. Hugo GD, Rosu M. Advances in $4 D$ radiation therapy for managing respiration: Part I - 4D imaging. Z Med Phys. 2012;22:258-71.

30. Yoganathan SA, Maria Das KJ, Mohamed Ali S, Agarwal A, Mishra SP, Kumar S. Evaluating the four-dimensional cone beam computed tomography with varying gantry speed. Br J Radiol. 2016;89:20150870.

31. Cooper BJ, O'Brien RT, Balik S, Hugo GD, Keall PJ. Respiratory triggered 4D cone-beam computed tomography: a novel method to reduce imaging dose. Med Phys. 2013;40:041901.

32. Abuhaimed A, Martin CJ, Sankaralingam M, Gentle DJ. A Monte Carlo investigation of cumulative dose measurements for cone beam computed tomography (CBCT) dosimetry. Phys Med Biol. 2015;60:1519-42.

33. Li T, Xing L, Munro P, McGuinness C, Chao M, Yang Y, et al. Fourdimensional cone-beam computed tomography using an on-board imager. Med Phys. 2006;33:3825-33.

34. McMillan K, McNitt-Gray M, Ruan D. Development and validation of a measurement-based source model for kilovoltage cone-beam CT Monte Carlo dosimetry simulations. Med Phys. 2013;40:111907.

\section{Submit your next manuscript to BioMed Central and we will help you at every step:}

- We accept pre-submission inquiries

- Our selector tool helps you to find the most relevant journal

- We provide round the clock customer support

- Convenient online submission

- Thorough peer review

- Inclusion in PubMed and all major indexing services

- Maximum visibility for your research

Submit your manuscript at www.biomedcentral.com/submit
Biomed Central 